Preprint typeset in JHEP style - HYPER VERSION

DAMTP-2007-79

\title{
Scanning the Landscape of Flux Compactifications: Vacuum Structure and Soft Supersymmetry Breaking
}

\author{
Shehu S. AbdusSalam, Joseph P. Conlon, Fernando Quevedo, Kerim Suruliz \\ DAMTP, Centre for Mathematical Sciences, \\ Wilberforce Road, Cambridge, CB3 0WA, UK. \\ E-mails: s.s.abdussalam@damtp.cam.ac.uk, j.p.conlon@damtp.cam.ac.uk, \\ f.quevedo@damtp.cam.ac.uk, k.suruliz@damtp.cam.ac.uk
}

\begin{abstract}
We scan the landscape of flux compactifications for the Calabi-Yau manifold $\mathbb{P}_{[1,1,1,6,9]}^{4}$ with two Kähler moduli by varying the value of the flux superpotential $W_{0}$ over a large range of values. We do not include uplift terms. We find a rich phase structure of AdS and dS vacua. Starting with $W_{0} \sim 1$ we reproduce the exponentially large volume scenario, but as $W_{0}$ is reduced new classes of minima appear. One of them corresponds to the supersymmetric KKLT vacuum while the other is a new, deeper non-supersymmetric minimum. We study how the bare cosmological constant and the soft supersymmetry breaking parameters for matter on $\mathrm{D} 7$ branes depend on $W_{0}$, for these classes of minima. We discuss potential applications of our results.
\end{abstract}


-TOC- Contents

1. Introduction 1

2. Moduli Fixing and Soft Terms 2

3. The $\mathbb{P}_{[1,1,1,6,9]}^{4}$ Model

3.1 Analytic Results 5

4. Structure of the Vacua Sets 8

4.1 Dependance on non-Perturbative Effects: $a$ and $b$ Parameters 8

4.1.1 Case $a \leq b$

4.1.2 Case $a>b$

4.2 Dependence on Fluxes: $W_{0}$ Parameter 12

4.2.1 Extremely small $W_{0}, W_{0} \leq W_{\text {crit }, 1}$

4.2.2 Small $W_{0}, W_{\text {crit }, 1} \leq\left|W_{0}\right| \leq W_{\text {crit }, 2}$

4.2.3 Small $W_{0}, W_{\text {crit }, 2} \leq\left|W_{0}\right| \leq W_{\text {crit }, 3}$

4.2.4 Large $W_{0}, W_{\text {crit }, 3} \leq\left|W_{0}\right|$

5. Soft Terms Structure in the Vacua Sets 14

5.1 D7 soft terms 14

6. Conclusions 15

\section{Introduction}

Originally it was hoped that, once non-perturbative effects were understood, string theory would have a unique vacuum. Finding this vacuum would allow all low-energy physical parameters to be directly computed. However, as our understanding developed this view has come to be seen as naive. There instead seems to be a landscape of many different vacua, each having different physical properties. Different vacua may have different gauge groups, different particle representations, and different values for the cosmological constant. The vacuum that we inhabit may be just one of a very large set of possibilities.

The construction that puts this problem in sharpest focus are flux compactifications, in which the RR and NS-NS fields present in string theory take non-trivial profiles in the vacuum (for reviews see [1]). Fluxes take integral charges within the co-homology lattice of the compactification manifold. As typical Calabi-Yaus may have $\mathcal{O}(100)$ cycles, the number of different profiles the fluxes can be given is exceedingly large. Each choice of fluxes correspond to a different vacuum state. As the fluxes enter the low-energy Lagrangian, for 
example through the dynamics of moduli fields, there exist very many different low energy Lagrangians.

The landscape of low-energy theories removes the hope of a unique determination of the vacuum state. However, it is fortunately not the case that the low-energy theories are entirely arbitrary. In IIB compactifications, the fluxes stabilise the dilaton and complex structure moduli through the superpotential [2, 3]:

$$
W=\int G_{3} \wedge \Omega
$$

However the direct effect of the fluxes on the dynamics of the Kähler moduli is relatively limited, and is mostly determined by the vev of the above flux superpotential. Different vacua have been identified depending on different values of the flux superpotential $W_{0}$. For example, when $\left|W_{0}\right| \sim 1$ there exist non-supersymmetric vacua at exponentially large values of the volume (the large volume models [0]) whereas for $W_{0} \ll 1$ there exist supersymmetric vacua at relatively small volume (the KKLT scenario [4]). These represent different phases of the landscape, and the location and properties of these phases depend on the values of the fluxes.

In this note we perform a systematic scan on the landscape for flux compactifications on the Calabi-Yau $\mathbb{P}_{[1,1,1,6,9]}^{4}$. This Calabi-Yau manifold provides one of the simplest concrete non-trivial examples of moduli stabilisation. It has been very much studied over the past few years [5, 6, 6] and has become a prototypical example for explicit calculations both for cosmological [8], phenomenological [9, 10, 11, 12, 13, 14, 15, 16] and astrophysical issues [17]. It is then interesting to fully explore the vacuum structure of this Calabi-Yau and contrast with the partial results found previously. In particular, the vacuum structure can be only fully explored numerically. We perform such an analysis here. We scan across values of $W_{0}$ ranging over approximately twenty orders of magnitude, studying the different phases and properties of the vacua that appear during this scan. Several features emerge, for example the existence at small volumes of a new class of vacua different from the standard KKLT and large volume minima. We expect that the rich vacuum structure of this manifold will also be shared in more complicated compactifications.

The organisation of this note is as follows. In section 2 we give a brief review of the effects that enter the moduli potential and the computation of soft supersymmetry breaking terms. In section 3 we describe the model we use $\left(\mathbb{P}_{[1,1,1,6,9]}^{4}\right)$ and the analytic results on vacuum structure that are available. In section 1 we describe the scan over $W_{0}$ and the structure of the different minima that are present. In section 0 we study how the soft terms vary as we scan over $W_{0}$, and in section 6 we conclude.

\section{Moduli Fixing and Soft Terms}

We work in the effective supergravity limit of type $I I B$ string theory [18]. Its massless bosonic fields in ten dimensions consist of the metric $g_{M N}$; two 2-forms $B_{M N}, C_{M N}$ with field strengths $H_{3}$ and $F_{3}$ respectively; a complex dilaton/axion scalar field

$$
S=e^{-\phi}+i a
$$


and a 4 -form $C_{4}$ with self-dual field strength. Upon compactification the $10 D$ background metric splits into a direct product of an $N=1$ supersymmetric $4 D$ Minkowski spacetime and a $6 D$ Calabi-Yau orientifold. The Calabi-Yau contains 2-cycles, their dual 4-cycles, and 3-cycles. The sizes of the 4-cycles define the Kähler moduli fields

$$
T_{i}=\tau_{i}+i \theta_{i}
$$

while those of the 3-cycles define the complex structure moduli fields $U_{a}$.

Fluxes thread the internal 3-cycles $\Sigma_{a}$ and are quantised in integral co-homology

$$
\int_{\Sigma_{a}} H_{3}=n_{a}, \quad \int_{\Sigma_{b}} F_{3}=m_{b}, \quad n_{a}, m_{b} \in \mathbb{Z}
$$

They generate a Kähler moduli independent superpotential [2]

$$
W=\int G_{3} \wedge \Omega
$$

where $G_{3}=F_{3}-i \mathrm{SH}_{3}$ and $\Omega$ is the holomorphic (3,0)-form of the internal space. This fixes the dilaton and complex structure moduli fields [3]. After adding non-perturbative effects involving the Kähler moduli, such as gaugino condensation, the effective superpotential takes the form [4]

$$
\hat{W}=W_{0}+\sum_{i} A_{i} e^{-a_{i} T_{i}} .
$$

s-pot

Here $W_{0}=\left\langle\int G_{3} \wedge \Omega\right\rangle$. Together with these the four-dimensional effective theory is specified by the Kähler potential at tree level

$$
\hat{K}=-2 \ln \mathcal{V}+K_{c s}
$$

where $\mathcal{V}$ is the Einstein-frame volume of the CY internal space and $K_{c s}$ carries the complex structure moduli and the dilaton dependence. It can be easily seen from here that the Kähler moduli can be fixed at supersymmetric $\left(D_{i} W=0\right)$ minima of the $\mathcal{N}=1$ supergravity (scalar) potential

$$
V=e^{\hat{K}}\left[\hat{K}^{i \bar{j}} D_{i} \hat{W} D_{\bar{j}} \overline{\hat{W}}-3|\hat{W}|^{2}\right]
$$

where

$$
D_{i} \hat{W}=\partial_{i} \hat{W}+\left(\partial_{i} \hat{K}\right) \hat{W} \text { and } \hat{K}^{i \bar{j}}=\left(\hat{K}_{i \bar{j}}\right)^{-1} .
$$

It is important to note that the fluxes (2.3) are quantised in units of the string scale $\alpha^{\prime}$ and so naturally $\left|W_{0}\right|$ takes $\mathcal{O}(1)$ values. But since general Calabi-Yau manifolds have large numbers of three cycles there are very many ways of turning on fluxes and by tuning the flux integers (2.3) it is possible for $\left|W_{0}\right|$ to take values arbitrarily close to zero [20]. ${ }^{1}$ The scanning performed in this note will vary $W_{0}$ as a smooth parameter. The Kähler

\footnotetext{
${ }^{1}$ It is however bounded above by an $\mathcal{O}(1)$ constant since the magnitudes of fluxes themselves are bounded by tadpole cancellation conditions.
} 
potential also receives $T_{i}$-dependent perturbative corrections that can be equally or more important in stabilisation compared to the non-perturbative corrections to $W$ [7, 9].

Supersymmetry breaking occurs if the moduli are fixed at non-supersymmetric minima such as the large-volume minimum. Supersymmetry breaking is quantified by the F-terms

$$
F^{m}=e^{\hat{K} / 2} \hat{K}^{m \bar{n}} D_{\bar{n}} \overline{\hat{W}}, \quad \hat{K}^{m \bar{n}}=\left(\partial_{m} \partial_{\bar{n}} \hat{K}\right)^{-1} .
$$

fterm

Given the F-terms for each class of minima the soft supersymmetry breaking terms are computed from the effective supergravity Lagrangian density first by expanding the potentials in powers of matter fields $C^{\alpha}$

$$
\begin{aligned}
W & =\hat{W}+\mu H_{1} H_{2}+\frac{1}{6} Y_{\alpha \beta \gamma} C^{\alpha} C^{\beta} C^{\gamma}+\ldots \\
K & =\hat{K}+\tilde{K}_{\alpha \bar{\beta}} C^{\alpha} C^{\bar{\beta}}+\left(Z H_{1} H_{2}+\text { h.c. }\right)+\ldots
\end{aligned}
$$

Here $H_{1,2}$ represent vector-like matter (in particular MSSM higgs bosons) and $\tilde{K}_{\bar{\alpha} \beta}$ is the Kähler metric for matter fields. Assuming a diagonal matter metric the Lagrangian density can be written as

$$
\mathcal{L}_{\text {soft }}=\tilde{K}_{\alpha} \partial_{\mu} C^{\alpha} \partial^{\mu} \bar{C}^{\bar{\alpha}}-m_{\alpha}^{2} C^{\alpha} \bar{C}^{\bar{\alpha}}-\left[\frac{1}{6} A_{\alpha \beta \gamma} \hat{Y}_{\alpha \beta \gamma} C^{\alpha} C^{\beta} C^{\gamma}+\ldots\right]
$$

with the scalar masses and A-terms given by 27]

$$
\begin{aligned}
m_{\alpha}^{2} & =m_{3 / 2}^{2}+V_{0}-F^{\bar{m}} F^{n} \partial_{\bar{m}} \partial_{n} \log \tilde{K}_{\alpha} \\
A_{\alpha \beta \gamma} & =F^{m}\left[\partial_{m} \hat{K}+\partial_{m} \log Y_{\alpha \beta \gamma}-\partial_{m} \log \left(\tilde{K}_{\alpha} \tilde{K}_{\beta} \tilde{K}_{\gamma}\right)\right] .
\end{aligned}
$$

The gravitino mass is given by

$$
m_{3 / 2}=e^{\hat{K} / 2}|\hat{W}|
$$

The canonically normalised gaugino masses are

$$
M_{a}=\frac{1}{2} \frac{F^{m} \partial_{m} f_{a}}{\operatorname{Re} f_{a}}
$$

where $f_{a}$ is the gauge kinetic function whose form depends on whether the gauge fields come from D3 or D7-branes: at tree level $f_{D 3}=S, f_{D 7}=T$. In the following section 3 we describe the Calabi-Yau manifold representing the internal space we use as an explicit model for the soft terms calculations.

\section{The $\mathbb{P}_{[1,1,1,6,9]}^{4}$ Model}

For concreteness we work with the well studied $\mathbb{P}_{[1,1,1,6,9]}^{4}$ Calabi-Yau compactification with two Kähler moduli fields: $T_{b}$ and $T_{s}$ with the first controlling the overall volume of the internal space and the second one corresponding to the size of a smaller 4-cycle that 
may be considered as a blow-up cycle. With the dilaton and complex structure moduli fixed by fluxes the effective theory for the Kähler moduli is

$$
\begin{aligned}
\hat{W} & =W_{0}+A e^{-a T_{s}}+B e^{-b T_{b}}, \\
\hat{K} & =-2 \log \left(\mathcal{V}+\frac{\xi}{2}\right), \\
\text { where } \mathcal{V} & =\frac{1}{9 \sqrt{2}}\left(\tau_{b}^{\frac{3}{2}}-\tau_{s}^{\frac{3}{2}}\right) .
\end{aligned}
$$

Here $T_{b, s}=\tau_{b, s}+i \theta_{b, s}$ are the Kähler moduli, $a, b=\frac{2 \pi}{N_{a, b}}$ for gaugino condensation on a gauge group of rank $N_{a, b}$ and $\xi$ parametrises the $\alpha^{\prime 3}$ correction to the Kähler moduli space. The scalar potential takes the form

$$
\begin{aligned}
V= & e^{\hat{K}} \sum_{i, j} K^{i \bar{\jmath}} a_{i} A_{i} a_{j} \overline{A_{j}} e^{-\left(a_{i} T_{i}+a_{j} T_{j}\right)} \\
& +\left(e^{\hat{K}} \sum_{i, j} K^{i \bar{\jmath}}\left(-a_{i} A_{i}\right) e^{-a_{i} T_{i}} \overline{\hat{W}\left(\partial_{j} \hat{K}\right)}+c . c .\right) \\
& +3 \xi e^{\hat{K}}|\hat{W}|^{2} \frac{\xi^{2}+7 \xi \mathcal{V}+\mathcal{V}^{2}}{(\mathcal{V}-\xi)(2 \mathcal{V}+\xi)^{2}} .
\end{aligned}
$$

scalarV

This potential is singular at $\mathcal{V}=\xi$ and therefore constraints the valid range of values of the volume to $\mathcal{V}>\xi$. We will scan over $W_{0}$ keeping all other parameters (including $g_{s}$ ) constant. In the full theory different choices of fluxes would also change the values of $A_{i}$ and $g_{s}$. The purpose of our restriction to scanning simply over $W_{0}$ is that it makes it easy to see the phase structure of minima without attempting to vary all scales at the same time.

\subsection{Analytic Results}

Depending on the values of flux the superpotential $W_{0}$ and the magnitude of the gaugino condensation parameters $a$ and $b$, different types of vacua are realised from the $\mathbb{P}_{[1,1,1,6,9]}^{4}$ model. Here we present the general results about the vacuum structure that can be derived analytically and leave the numerical results for the following section.

The supersymmetric minima are easiest to study analytically since they are found by solving the first order equations $D W=0$ for each of the moduli fields. For the two moduli case, we have

$$
D_{s} \hat{W}=D_{b} \hat{W}=0
$$

This implies:

$$
\begin{aligned}
& a A e^{-a T_{s}}-\frac{3 \hat{W}}{2 \mathcal{V}} \tau_{s}^{\frac{1}{2}}=0, \\
& b B e^{-b T_{b}}+\frac{3 \hat{W}}{2 \mathcal{V}} \tau_{b}^{\frac{1}{2}}=0 .
\end{aligned}
$$

These give an AdS solution with the constraint that

$$
W_{0}=e^{-a \tau_{s}}\left[1+\frac{2}{3} a \tau_{s}\right]-e^{-b \tau_{b}}-\frac{a \xi}{3 \tau_{s}^{1 / 2}} e^{-a \tau_{s}}
$$


at the minimum of the potential. The depth of the potential at the minimum is given by

$$
V_{0}=-\frac{4}{3} \frac{a^{2} e^{-2 a \tau_{s}}}{\tau_{s}}=-\frac{4}{3} \frac{b^{2} e^{-2 b \tau_{b}}}{\tau_{b}} .
$$

Taking the ratio of equations in (3.6) gives

$$
e^{\left(b T_{b}-a T_{s}\right)}=-\frac{b B}{a A}\left(\frac{\tau_{s}}{\tau_{b}}\right)^{1 / 2} .
$$

By absorbing the phases of $A$ and $B$ into $T_{s}$ and $T_{b}$ it can be seen that the angular phases $\theta_{s}, \theta_{b}$ are constrained to satisfy $e^{i a \theta_{s}}=-e^{i b \theta_{b}}$. Also from equations (3.6) it can be seen that the phase $e^{i a \theta_{s}}$ is the same as the phase of the flux superpotential $W_{0}$ which without loss of generality can be fixed to -1 so $\theta_{s}=\pi, \theta_{b}=0$. We are then left with the real parameters $\tau_{s}, \tau_{b}$ for which we have two real equations. It is straightforward to extract the following general conclusions from them.

1. There are no supersymmetric solutions with $a \ll b$ : This is easily seen by rewriting equation (3.9) into the form

$$
b \tau_{b}-a \tau_{s}=\log \left[\frac{b B}{a A}\left(\frac{\tau_{s}}{\tau_{b}}\right)^{1 / 2}\right] .
$$

This implies that $b \tau_{b} \sim a \tau_{s}$ at the minimum. But $\tau_{b}>\tau_{s}$ is required in order to get positive volume and therefore as long as $A$ and $B$ are comparable then $a>b$ must be satisfied. In the case of a large hierarchy between $A$ and $B$ supersymmetric solutions may exist with $a$ slightly less than $b$.

2. There are no non trivial supersymmetric solutions for $W_{0}=0$ : Using equations (3.6) and (3.9) above it can be see that for $W_{0}=0$ and $a>b$

$$
a b-\frac{3}{2 \mathcal{V}}\left[b \tau_{s}^{1 / 2}-a \tau_{b}^{1 / 2}\right]=0
$$

and substituting the expression for the volume from equation 3.3 gives

$$
a b\left(\tau_{b}^{3 / 2}-\tau_{s}^{3 / 2}\right)+3\left(a \tau_{b}^{1 / 2}-b \tau_{s}^{1 / 2}\right)=0 .
$$

This equation can never be satisfied because both terms are positive. The trivial case of getting a supersymmetric solution with $W_{0}=0$ is only possible at the decompactification limit.

It follows that the supersymmetric KKLT class of vacua exist only for $a>b$ and $W_{0} \neq 0$. In the one modulus case it was clear that $W_{0}=0$ provides no solutions since a single exponential superpotential gives a runaway behaviour, but in the many Kähler moduli case this is not automatic because the sum of exponentials in $W$ may have been in principle enough to generate a nontrivial vacuum. Furthermore, as for the one modulus case, the supersymmetric KKLT solutions can exist only as long as $W_{0}$ is small enough that equations (3.6) may be solved with large enough $\tau_{s}, \tau_{b}$ to keep the supergravity approximations valid. 
As from equations (3.6) it follows that $\tau_{b} \sim \ln W_{0}, \tau_{s} \sim \ln W_{0}$, as $W_{0} \rightarrow 0$ the behaviour of the volume and potential with $W_{0}$ is

$$
\mathcal{V} \sim\left(-\ln W_{0}\right)^{3 / 2}, \quad V_{0}=-3 W_{0}^{2}
$$

Besides the supersymmetric minimum $M_{K L}$, the scalar potential has another class of

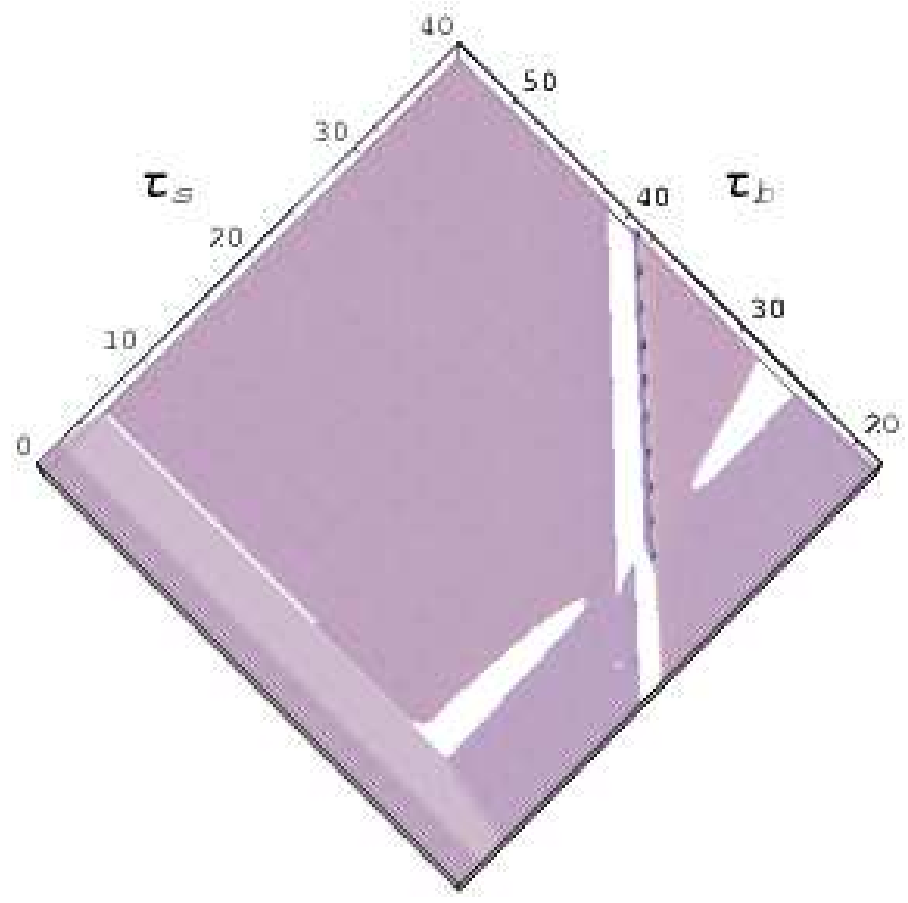

valleys

Figure 1: A 3D plot of $\log$ of the potential (3.4) versus the two moduli fields $\tau_{b, s}$. On either side of the $\tau_{b}=\tau_{s}$ white patch there are patches representing regions where the potential energy is negative and hence a valley where a minimum may be found. Any minimum in the valley to the right of $\tau_{b}=\tau_{s}$ is unphysical since it has $\mathcal{V}<0$. The $\mathcal{M}_{L V}$ minima set is found in the vertical part of the almost L-shaped valley. The other minima sets, $\mathcal{M}_{K L}$ and $\mathcal{M}_{\text {new }}$, are found in the horizontal part of the L-valley.

minimum $\mathcal{M}_{L V}$ which has been found analytically. It corresponds to non-supersymmetric AdS and occurs at exponentially large volume with $\theta_{s}=0$ and $\tau_{b} \gg \tau_{s}$. In the large volume approximation the potential has the form

$$
V=\sum_{i, j} C_{1} \frac{\sqrt{\tau_{s}} e^{-2 a \tau_{s}}}{\mathcal{V}}-\sum_{i} C_{2} \frac{\tau_{s} e^{-a \tau_{s}}}{\mathcal{V}^{2}}+\frac{C_{3}}{\mathcal{V}^{3}}
$$

The third term in (3.14) results from the $\alpha^{\prime}$ correction to the Kähler potential equation(2.6) and the $C$ 's are constants of order unity. It can be seen [7, 9] that $V$ has a minimum such that one of the moduli, $\tau_{b}$, is exponentially large and the other is $\tau_{s} \gtrsim \mathcal{O}(1)$. The volume at the minimum is given by

$$
\mathcal{V} \sim W_{0} e^{a \tau_{s}}
$$

For generic values of the flux superpotential $W_{0} \sim 1$ this volume is exponentially large. 
In the sections that follow we will be more explicit concerning the concrete range of parameters in which the supersymmetric AdS and the non supersymmetric AdS minima exist. We will also demonstrate numerically the existence of a new class $M_{n e w}$ of nonsupersymmetric AdS and dS vacua in particular ranges of $W_{0}$ values. The de Sitter vacua obtained are analogous to those obtained in [29] (see also [22, 23]).

The plot in figure 1 gives an instance of all three minima coexisting.

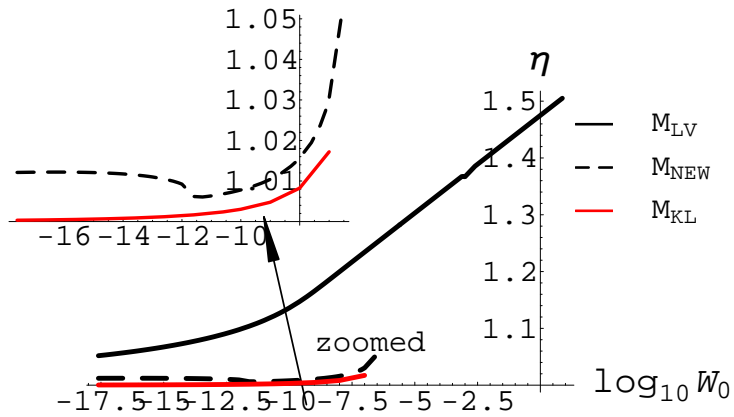

susybrk

Figure 2: Check for supersymmetry breaking or preservation in the three classes of vacua. Supersymmetry is preserved if the ordinate $\eta=\left|V_{0} / 3 m_{3 / 2}^{2}\right|$ is exactly equal to 1 . The solid black line represents the $\mathcal{M}_{L V}$ set in which clearly supersymmetry is broken for all $W_{0}$. The red line is the KKLT minima set $\mathcal{M}_{K L}$ and its non-supersymmetric extension. The dashed lines correspond to the $\mathcal{M}_{\text {new }}$ set of minima. Supersymmetry is always broken here also but at a smaller scale than for $\mathcal{M}_{\mathcal{L V}}$.

\section{Structure of the Vacua Sets}

minstruc

Depending on the non-perturbative and flux contributions multiple minima can coexist. The minima are differentiated by the volume of the internal space $\mathcal{V}$, the magnitude of the bare cosmological constant $V_{0}$, the value of the axionic phase $\theta$ at which the minimum settles and whether supersymmetry breaking occurs or not. In order to study the variation of these quantities with different flux choices we scan over the minima of the scalar potential

eqn(3.4) over different values of $W_{0}$. In addition to this continuous scanning there is also a discrete parameter choice, that heavily affects the structure of the minima, coming from the relative magnitudes of the parameters $a$ and $b$. For the numerical estimates we have chosen:

$$
A=B=1, \quad \xi=1.31, \quad a=2 \pi / 0.85
$$

and, depending on whether the case $a=b$ or $a>b$ is considered, $b=\{2 \pi / 0.85,2 \pi / 3.85\}$.

\subsection{Dependance on non-Perturbative Effects: $a$ and $b$ Parameters}

We address the two cases $a>b$ and $a<b$ separately. 


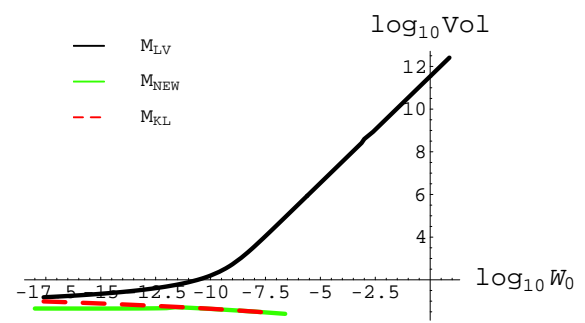

Figure 3: Plot shows the internal volume structure with various $W_{0}$ values in each of $\mathcal{M}_{L V}$ (black curve), $\mathcal{M}_{K L}$ (red line) and $\mathcal{M}_{\text {new }}$ vacua sets. The transition from a large to small volume

grav1

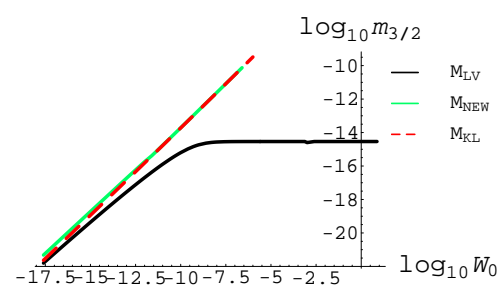

Figure 4: This plot shows the gravitino mass variation with $W_{0}$ for all the three vacua sets. In small $W_{0}$ regions corresponding to the small volume phase the mass is the same in all the minima types.

aleqb

\subsubsection{Case $a \leq b$}

As already shown in section 3.1 no supersymmetric minimum exists for these values of the parameters $a \leq b$. Scanning across $W_{0}$, the only type of minima of the scalar potential found corresponds to the set $\mathcal{M}_{L V}$ containing the large-volume minimum. In the $W_{0} \sim \mathcal{O}(1)$ region where the volume is exponentially large, the scaling of the volume goes as $\mathcal{V} \sim W_{0} e^{a \tau_{s}}$. As $W_{0}$ is decreased, the volume decreases linearly. This continues until $W_{0}$ is sufficiently small to compensate the large exponential, such that the volume approaches unity. As $W_{0}$ is further decreased, the minimum does not disappear but instead exists in a small-volume phase. As far as we have been able to check, this minimum continues to exist down to arbitrarily small values of $W_{0}$. The variation of the volume with $W_{0}$ in this minima set compared with the behaviour in the other sets (described in section 4.1.2) is shown in figure 3. In the large-volume region, the behaviour $\mathcal{V} \sim W_{0}$ is clear. After the transition to the small-volume region, the internal volume is seen to be essentially constant and insensitive to further decreases in $W_{0}$. It is possible to understand analytically why these smaller volume minima exist in the $\mathcal{M}_{L V}$ set at very small values of $W_{0}$. The largevolume construction ensures that the potential goes to zero from below at infinite volumes. However, the $\alpha^{\prime 3}$ term in the scalar potential diverges positively at small volumes. As the potential goes to positive infinity at small volumes, and approaches zero from below at large volumes, a minimum must exist somewhere in the intermediate regime. Thus decreasing $W_{0}$ brings the large-volume minimum to smaller values but does not destroy its existence.

The gravitino mass $m_{3 / 2}$ determines the overall scale of the supersymmetry breaking parameters. Figure 14 shows the variation of the gravitino mass with $W_{0}$ in this minima set compared with the behaviour in the other sets (described in section 4.1.2). The shape of the curve is as expected for the large-volume minima: in the large volume limit $m_{3 / 2}=\frac{W_{0}}{\mathcal{V}}$ and $\mathcal{V} \sim W_{0}$, making the gravitino mass independent of $W_{0}$. Once $W_{0}$ is sufficiently small, the volume of the minimum remains fixed and small. However $W_{0}$ continues to decrease and thus the gravitino mass decreases linearly with $W_{0}$.

The transition from large volume to small volume phases is best seen from the structure 
of the cosmological constant with $W_{0}$ in the $\mathcal{M}_{L V}$ vacua set compared with the behaviour in the other sets (described in section 4.1.2) as shown in figures 5 and 6 . The figures clearly show the existence of a minimal value for the cosmological constant around $W_{0} \sim 10^{-9}$. This feature can be analytically explained: For larger values of $W_{0}$, we are in the largevolume region for which the minima can be found analytically with an AdS cosmological constant

$$
V_{0} \sim \frac{W_{0}^{2}}{\mathcal{V}^{3}} \sim \frac{1}{W_{0}}
$$

As $W_{0}$ decreases, $\left|V_{0}\right|$ therefore increases linearly, as indeed seen in the figure 5 . Once in the small volume region, $\mathcal{V}$ no longer has significant dependence on $W_{0}$, and so

$$
V \sim \frac{W_{0}^{2}}{\mathcal{V}^{3}} \sim W_{0}^{2}
$$

so the magnitude of $V_{0}$ decreases quadratically with $W_{0}$. This change is seen in figure 6 through the change in slope and direction. These minima are non-supersymmetric for all values of $W_{0}$.

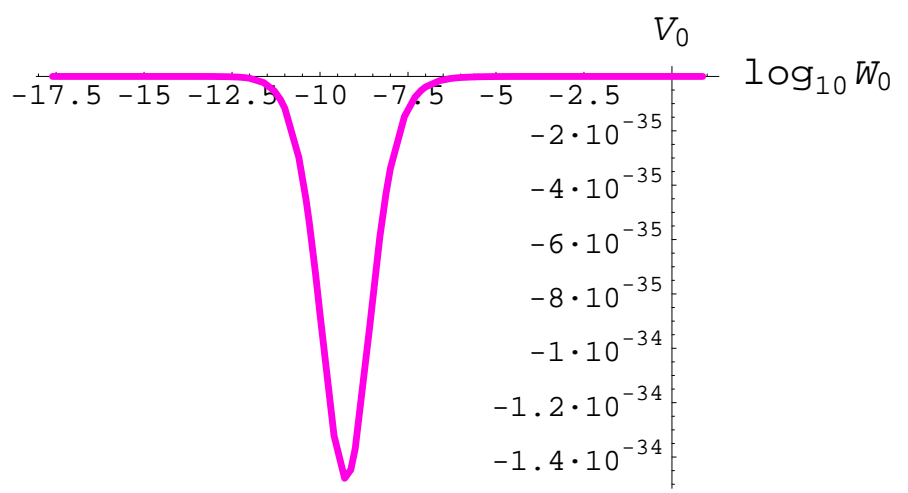

$\operatorname{vacc1}$

Figure 5: The variation of the bare cosmological constant $V_{0}$ with $W_{0}$ for the large-volume minimum. The minimum value of $V_{0}$ lies around $W_{0} \sim 10^{-9}$ and marks the phase transition.

\subsubsection{Case $a>b$}

In the $a>b$ scenario the potential eqn(3.4) has greater richness of minima. Four distinct classes of vacua can be identified for different values of $W_{0}$. We enumerate these and describe their properties:

1. The large-volume minima $\mathcal{M}_{L V}$. These are realised for sufficiently large values of $W_{0}$ and have a similar structure to the case $a \leq b$ of the previous section. $\tau_{s}$ is stabilised by effects non-perturbative in $\tau_{s}$ and $\tau_{b}$ is stabilised by effects perturbative in $\tau_{b}$. With the superpotential parameters $A, B$ real and positive and $W_{0}$ real and negative the minima settle on the at $\theta_{s}=0$ with $\theta_{b}$ undetermined up to non-perturbative effects that play no role in stabilising $\tau_{b}$. 


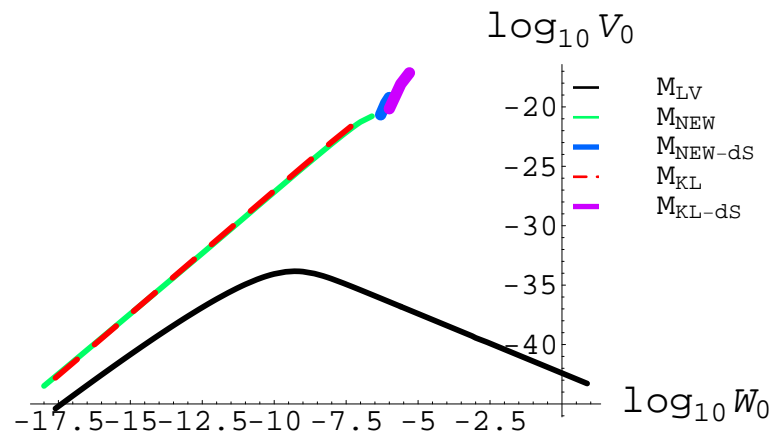

vacc

Figure 6: This plot shows the structure of $V_{0}$ over $W_{0}$ in the three vacua sets. The depth of the potential at the different minima or the cosmological constant is identical for the $\mathcal{M}_{K L}$ and $\mathcal{M}_{\text {new }}$ vacua set. The minima in these sets become de Sitter in a small interval of $W_{0}$ values. The depth of the potential (now positive) in this region is shown by the bolder lines. The black curve is a log-log plot of $V_{0}$ for the $\mathcal{M}_{L V}$ vacua set. The slopes on either side of the turning point, +2 and -1 , show the different structure of $V_{0}$ on $W_{0}$ from large and small volume regions.

2. The supersymmetric (KKLT) minimum [⿶], which exists at small volume $\left(\mathcal{V} \sim \ln W_{0}\right)$ for sufficiently small values of $W_{0}$. It has

$$
a \tau_{s} \sim \ln W_{0}, \quad b \tau_{b} \sim \ln W_{0} .
$$

Here $\tau_{s}$ is stabilised by effects non-perturbative in $\tau_{s}$ and $\tau_{b}$ is stabilised by effects non-perturbative in $\tau_{b}$. With the above choice of sign conventions, $\theta_{s}=\pi$ and $\theta_{b}=0$.

3. A new set of minima $\mathcal{M}_{\text {new }}$ coexisting in the space of parameters with the supersymmetric $\mathcal{M}_{K L}$ with $\theta_{b}=0$ and $\theta_{s}$ is undetermined. This set has some similar properties to $\mathcal{M}_{K L}$ but is always non-supersymmetric as shown in figure 2. The structures of gravitino mass, the internal space volume and the cosmological constant $V_{0}$ in these sets of minima are similar as can be seen in the corresponding plots of the mentioned quantities in figures 3, 4 and 6. The minima settle such that

$$
b \tau_{b} \sim \ln W_{0}, \quad \ln W_{0} \ll a \tau_{s}, \quad \tau_{s}<\tau_{b} .
$$

$\tau_{b}$ is stabilised by effects non-perturbative in $\tau_{b}$, whereas $\tau_{s}$ is stabilised by effects perturbative in $\tau_{s}$. The origin of this kind of minima can be understood as follows. Suppose we first neglect the existence of the $\alpha^{\prime 3}$ corrections, and also take $\tau_{s}$ to be sufficiently large that the terms non-perturbative in $\tau_{s}$ can be ignored. In this case the only terms contributing to the scalar potential are those non-perturbative in $\tau_{b}$. In this limit the effective scalar potential is

$$
V=\frac{\tau_{b}^{2} a_{b}^{2} A_{b}^{2} e^{-2 a_{b} \tau_{b}}}{\mathcal{V}^{2}}-\frac{\tau_{b} a_{b} A_{b} W_{0} e^{-a_{b} \tau_{b}}}{\mathcal{V}}
$$

With this $\tau_{b}$ is fixed as in KKLT to generate an effective negative potential,

$$
V \sim-\frac{W_{0}^{2}}{\mathcal{V}^{2}}
$$


The volume is a function both of $\tau_{s}$ and $\tau_{b}$ and is thus not stabilised (as $\tau_{s}$ was not stabilised). This potential can be decreased by decreasing the volume. To stabilise $\tau_{s}$ the $\alpha^{\prime}$ correction has to be turned on. The appearance of the volume in the potential therefore generates a potential for $\tau_{s}$, which starts increasing (so as to decrease the volume and thus the potential). However, the $\alpha^{\prime 3}$ correction diverges positively at small volume, giving a total potential

$$
V \sim-\frac{W_{0}^{2}}{\mathcal{V}^{2}}+\frac{\xi W_{0}^{2}}{\mathcal{V}^{2}(\mathcal{V}-\xi)}
$$

so at some point this potential must turn around at small volume giving a nonsupersymmetric minima at small volume. As this minimum exists at very small volume (significantly smaller than in KKLT), it is not clear that the supergravity arguments leading to its existence can be trusted in the full string theory.

4. A de Sitter (dS) set of minima contained in the $\mathcal{M}_{K L}$ vacua set that are connected with other supersymmetric (KKLT) minima since one can move from the former to the latter set (and vice versa) by smoothly varying $W_{0}$. These are the 2 -modulus versions of the de Sitter minima of [29]. They exist for a very small range of $W_{0}$, where perturbative and non-perturbative corrections balance each other, in both $\mathcal{M}_{K L}$ and $\mathcal{M}_{\text {new }}$ vacua sets.

\subsection{Dependence on Fluxes: $W_{0}$ Parameter}

Varying the flux superpotential $W_{0}$ four distinct ranges with different vacua structure can be identified. These ranges are illustrated schematically in figure 7 and explained in the remaining part of this subsection.

\subsubsection{Extremely small $W_{0}, W_{0} \leq W_{\text {crit }, 1}$}

For extremely small values of $W_{0} \leq W_{\text {crit }, 1}$ there exist only two vacua sets of the potential eqn(3.4): $\mathcal{M}_{K L}$ and $\mathcal{M}_{\text {new }}$. Both are AdS, with the KKLT set being supersymmetric and the new set being non-supersymmetric. The potential in these minima sets essentially settles at the same depth. We define this range as region I.

\subsubsection{Small $W_{0}, W_{\text {crit }, 1} \leq\left|W_{0}\right| \leq W_{\text {crit }, 2}$}

Starting with the two minima set of the previous region I and as $W_{0}$ is increased to $W_{c r i t, 1} \sim 10^{-17}$ a third minima type appears. This corresponds to the non-supersymmetric large-volume minima set $\mathcal{M}_{L V}$. It is distinguished from the other ones by the $\theta_{s}$ axion phase. Initially the minima in this class appear at values of $\tau_{s}$ and $\tau_{b}$ very similar to those in $\mathcal{M}_{K L}$. But as $W_{0}$ is further increased, the volume rapidly increases and thus the minima separate leaving the other ones in the small $\left(\tau_{s}, \tau_{b}\right)$ region.

\subsubsection{Small $W_{0}, W_{\text {crit }, 2} \leq\left|W_{0}\right| \leq W_{\text {crit }, 3}$}

$W_{c r i t, 2}$ is defined as the maximal value of $W_{0}$ for which supersymmetric solutions are possible. As $W_{0}$ is increased beyond this point all the minima are non-supersymmetric because the equation $D_{i} W=0$ can no longer be solved. 


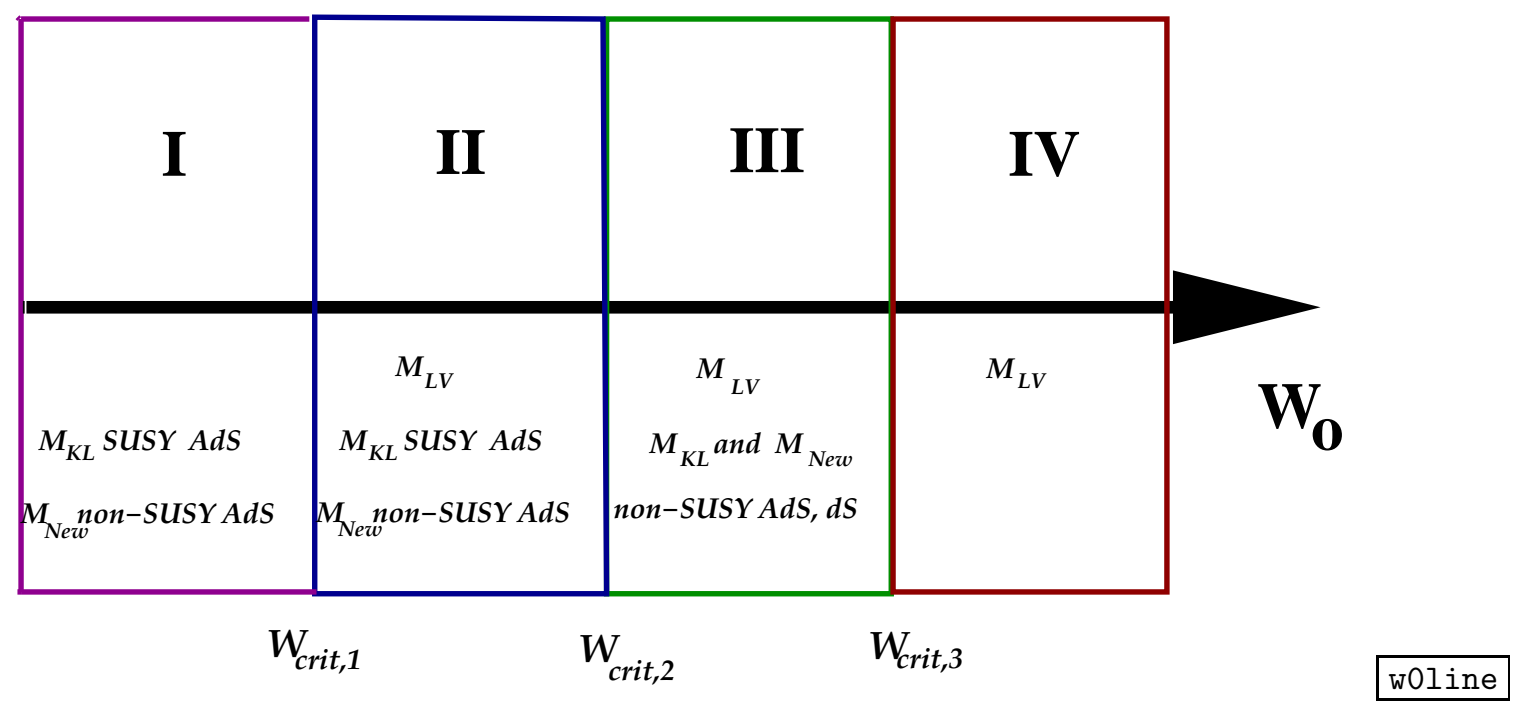

Figure 7: Four $W_{0}$ regions with different vacua sets for $a>b$. The large-volume set $\mathcal{M}_{L V}$ is the most generic, it is always non-supersymmetric AdS and disappears after 'merging' with the supersymmetric $\mathcal{M}_{K L}$ set at very small values of $W_{0}$. The KKLT set in $\mathcal{M}_{K L}$ and the new $\mathcal{M}_{\text {new }}$ set exist in the regions with very small $W_{0}$ and evolve into non-supersymmetric, AdS and then dS minima before disappearing altogether for larger values of $W_{0}$ as described in text (section 4.1.2).

For our model with two moduli fields

$$
D_{i} W=0 \rightarrow W_{0}=-\left[\sum_{j=s, b} A_{j} e^{-a_{j} T_{j}}+\frac{(\mathcal{V}+\xi / 2)}{t^{i}} a_{i} A_{i} e^{-a_{i} T_{i}}\right]
$$

where we have used $\partial_{T_{i}} K=-t^{i} /(\mathcal{V}+\xi / 2)$, where $t^{i}$ measures the area of 2-cycles with

$$
\tau_{k}=R e\left(T_{k}\right)=\partial_{t^{k}} \mathcal{V}=\frac{1}{2} \kappa_{i j k} t^{i} t^{j}, \quad \mathcal{V}=\frac{1}{6} \kappa_{i j k} t^{i} t^{j} t^{k}
$$

Note also that we require $\mathcal{V}>\xi>0$ so $t^{i} \neq 0$. From eqn(4.4) $t^{i} \rightarrow 0$ will imply $W_{0} \rightarrow \infty$ and hence supersymmetry cannot be preserved. For the symmetry to be preserved $t^{i}$ must be bounded away from zero. Hence for any supersymmetric minimum the possible $W_{0}$ values are bounded from above, $W_{0} \leq W_{\max }$, since larger $W_{0}$ require smaller $t^{i}$. In this manner any minimum with $W_{0}>W_{\max }$ will be non-supersymmetric. This explains why supersymmetry is broken in $\mathcal{M}_{K L}$ for $W_{0} \gtrsim W_{\text {max }}=W_{\text {crit }, 2}$.

As $W_{0}$ is further increased, the minima in the $\mathcal{M}_{K L}$ and $\mathcal{M}_{n e w}$ vacua set become de Sitter before disappearing altogether at $W_{0} \sim W_{\text {crit }, 3}$. As shown in figure 8 the minimum disappears when the positive term in $V$ becomes dominant. The range of $W_{0}$ over which the minima go de Sitter without introducing uplifting effects is rather small.

\subsubsection{Large $W_{0}, W_{c r i t, 3} \leq\left|W_{0}\right|$}

For $W_{0} \gtrsim W_{c r i t, 3}=2.5 \times 10^{-6}$ only minima in the large-volume phase $\mathcal{M}_{L V}$ survive. The small-volume phases and all other vacua type no longer exist.

The soft term structure is described in the next section 5 . 


\section{$V_{0}$ against $\tau_{5}$}

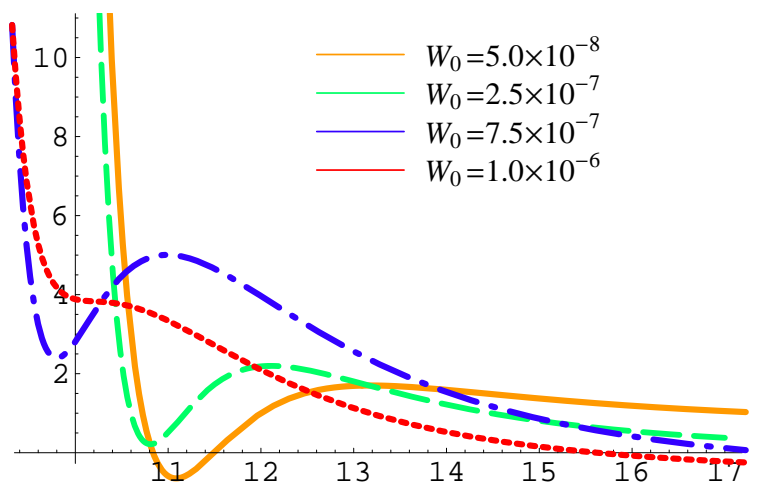

ds1to4

Figure 8: The behaviour of a minimum which becomes $d S$ before finally disappearing altogether as $W_{0}$ is increased in the range $W_{\text {crit }, 3}>W_{0}>W_{\text {crit }, 2}$.

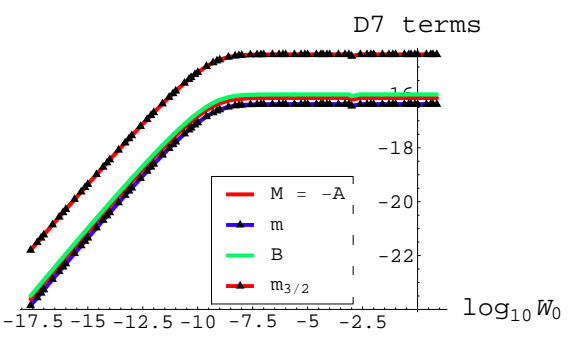

Figure 9: The dependence of soft terms for chiral D\%-brane matter on $W_{0}$ for $\mathcal{M}_{L V}$. The terms are degenerate and suppressed with respect to the gravitino mass. The amount of supression

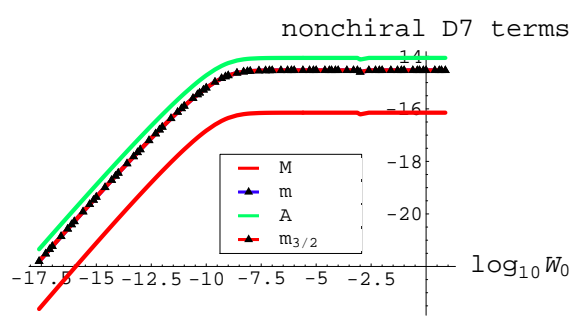

Figure 10: Structure of soft terms for nonchiral D\%-brane matter for $\mathcal{M}_{L V}$. The gaugino mass is reduced with respect to, while the scalar mass is degenerate with the gravitino mass. The A-term is slightly greater than $m_{3 / 2}$. Unlike the D3 soft terms, the small hierarchy between the d7-2 terms remains constant for all $W_{0}$.

\section{Soft Terms Structure in the Vacua Sets}

In this section we present the behaviour of soft terms as $W_{0}$ is varied. As in the $a \leq b$ case there is only one class of vacua, the $\mathcal{M}_{L V}$ set, which is also present in the $a \geq b$ case. Therefore, we only address the latter $(a>b)$ case. We focus on D7 soft terms as these are more phenomenologically relevant.

\subsection{D7 soft terms}

For matter fields in the bifundamental representation, arising from open strings stretching between magnetised D7-branes, the soft terms have been computed in [14 for the largevolume model case of $W_{0} \sim \mathcal{O}(1)$ as $m: M: A \sim 1: \sqrt{3}:-1$ with $m \sim m_{3 / 2}$. To obtain this result it was assumed that the D7 branes carrying the MSSM are wrapping the small 4-cycle, which we will also assume here. For the large volume phase, this is required to 


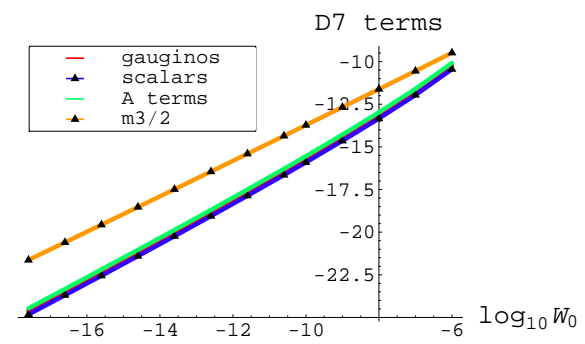

Figure 11: The dependence of soft terms for chiral D\%-brane matter on $W_{0}$ for $\mathcal{M}_{K L}$. Here the behaviour is different from the $\mathcal{M}_{L V}$ caßatus4 the soft terms scale as the gravitino mass with

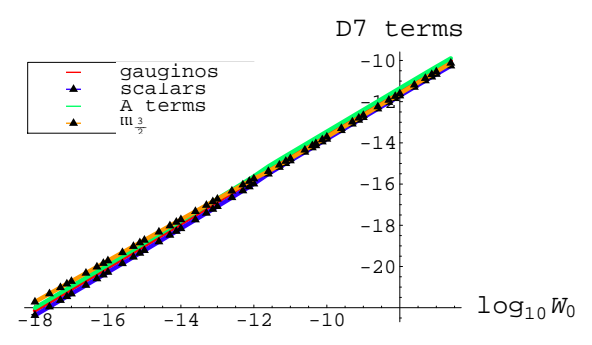

Figure 12: Structure of soft terms for nonchiral D\%-brane matter for $\mathcal{M}_{\text {new }}$. In this case all the soft terms scale as the gravitino mass.

d7-3 $W_{0}$.

obtain gauge couplings of the correct magnitude, since branes wrapping the large 4-cycle would yield gauge couplings far too small to be compatible with the observed values.

The numerically obtained soft terms structure in all of $\mathcal{M}_{L V}, \mathcal{M}_{K L}$ and $\mathcal{M}_{\text {new }}$ vacua sets over the different $W_{0}$ ranges is shown in figure 9. There are basically two different soft terms characteristics in $\mathcal{M}_{L V}$ over all the scanned $W_{0}$ values. Above the turning point $W_{0}^{c} \sim 10^{-9}$ the soft terms are independent of $W_{0}$. Below this critical value the soft terms vary linearly with $W_{0}$. In both cases the mass hierarchy between the soft terms $m, M, A$ and the gravitino is as predicted in [12, 14] for the large-volume model. The kind of mass hierarchy obtained is different for the case of D7 matter fields in the adjoint representation, as shown in figure 10. The main difference is that here only the gaugino mass term is suppressed compared to the gravitino mass. This is due to the fact that the F-term of the small Kähler modulus, $F^{s}$, is suppressed with respect to the gravitino mass by a factor $\log \left(M_{P} / m_{3 / 2}\right)$ as described in 12$]$.

For both the $\mathcal{M}_{K L}$ and $\mathcal{M}_{\text {new }}$ sets the soft terms for chiral matter scale with $W_{0}$ very different from the $\mathcal{M}_{L V}$ models as it can be seen in figures 11 and 12. For $\mathcal{M}_{K L}$ the gravitino mass is hierarchicaly larger than all the soft terms, but the difference increases slightly with decreasing $W_{0}$, whereas for $\mathcal{M}_{\text {new }}$ all the soft terms are degenerate with the gravitino mass.

\section{Conclusions}

In this paper we have performed, for a particular Calabi-Yau, a scan over the different phases of the landscape by varying $W_{0}$. We have varied $W_{0}$ by twenty orders of magnitude, from $\mathcal{O}(1)$ to $\mathcal{O}\left(10^{-20}\right)$, and have studied the structure and properties of the various minima.

A total of four different classes of minima were observed in the scan. These minima can be either supersymmetric or non-supersymmetric, dS or AdS, and at large and small volume. The supersymmetry breaking scales can be either high or low. We have plotted the variation of physical quantities such as the gravitino mass or the cosmological constant 
with $W_{0}$, and have been able to give analytic explanations for its behaviour. We have seen that certain minima can only exist in a restricted range of parameter space. For example, the large volume solution can cease to be present at sufficiently small values of $W_{0}$, turning into the KKLT solution, whereas the non-supersymmetric de Sitter minima analogous to those found in 29] are only present for a small range of $W_{0}$.

Some implications and potential applications of our results may be considered

1. Our mostly numerical analysis complements the analytical results that have been obtained before for this Calabi-Yau. It reproduces them in the regimes where the analytical results are valid but it uncovers previously unnoticed facts in the regimes where only the numerical analysis is available. Examples are the existence of the new class of non-supersymmetric AdS and dS minima and the soft terms behaviour for the large volume class in the domain of small flux superpotential. We expect a similar structure of minima to be present in more general Calabi-Yau compactifications.

2. The minima which are more reliable in this effective field theory analysis are the ones corresponding to very large volume. It is reassuring that in a large region of the landscape the soft supersymmetry breaking parameters remain constant and then their value can be considered generic within the landscape.

3. The KKLT and new minima exist for very small values of $W_{0}$ (and only for $a>b$ ) and are therefore less generic. They are also less reliable as long as the volume is relatively small, especially in the non-supersymmetric cases. Nevertheless, their existence within the effective field theory leads naturally to potential implications. Being non-supersymmetric, the new minima offer a pattern of soft supersymmetry breaking terms much different form the well studied large volume case (and the KKLT case once lifting is included). The change from supersymmetric AdS to nonsupersymmetric AdS to dS was already noted for the one modulus case in [29]. Here we have seen that the new minima follow a similar behaviour. It may be interesting to explore the cosmological implications and vacuum transitions of this structure especially in regimes where the three classes of minima coexist once a lifting is also included.

4. For the large volume minima in the regime where $W_{0}$ is small enough to cancel the large exponential dependence of the volume, the phenomenological implications are very different from the actual large volume regime that has been studied in the literature. As figure 3 illustrates, the gravitino mass stops being constant at a critical $W_{0}=W_{c}$ and then decreases with decreasing $W_{0}$. This allows to consider phenomenological scenarios where the string scale (independent of $W_{0}$ ) is large but the gravitino mass is a $\mathrm{TeV}$ (similar to the KKLT case studied in [28]). It may be interesting to explore the low-energy structure of soft terms for 'benchmark points' corresponding to the domain where $W \ll W_{c}$ and also at $W \sim W_{c}$ which will have distinctive physical behaviour than the $W>W_{c}$ studied already in detail all the way to LHC energies (see for instance [14, 31]). 
Our results lend support to the notion that the string landscape has a rich structure of vacua with different physical properties, and makes more pressing the task of determining which vacua have the necessary properties to be phenomenologically successful.

\section{Acknowledgments}

We thank B. Allanach, C. Burgess, M. Cicoli, D. Cremades, F. Denef, F. Feroz, M. P. Garcia del Moral, D. Grellscheid, S. Kom and D. Mackay for discussions. SA is supported by Gates Cambridge Scholarship Trust and a member of St Edmunds College, Cambridge. JC and KS are funded by Trinity College, Cambridge. FQ is partially funded by SFC and a Royal Society Wolfson Merit Award.

\section{References}

fluxcomp [1] M. Graña, Phys. Rept. 423, 91 (2006) [arXiv:hep-th/0509003]; M. R. Douglas and S. Kachru, arXiv:hep-th/0610102; F. Denef, M. R. Douglas and S. Kachru, arXiv:hep-th/0701050.

GVW [2] S. Gukov, C. Vafa and E. Witten, Nucl. Phys. B 584, 69 (2000) [Erratum-ibid. B 608, 477 (2001)] [arXiv:hep-th/9906070];

GKP [3] S. B. Giddings, S. Kachru and J. Polchinski, Phys. Rev. D 66, 106006 (2002) [arXiv:hep-th/0105097].

kklt [4] S. Kachru, R. Kallosh, A. Linde and S. P. Trivedi, Phys. Rev. D 68 (2003) 046005 [arXiv:hep-th/0301240].

[5] P. Candelas, A. Font, S. H. Katz and D. R. Morrison, Nucl. Phys. B 429 (1994) 626 [arXiv:hep-th/9403187].

[6] F. Denef, M. R. Douglas and B. Florea, JHEP 0406 (2004) 034 [arXiv:hep-th/0404257].

[7] V. Balasubramanian, P. Berglund, J. P. Conlon and F. Quevedo, JHEP 0503 (2005) 007 [arXiv:hep-th/0502058];

racetrack2

[8] J. J. Blanco-Pillado et al., JHEP 0609 (2006) 002 [arXiv:hep-th/0603129].

[9] J. P. Conlon, F. Quevedo and K. Suruliz, JHEP 0508 (2005) 007 [arXiv:hep-th/0505076].

[10] K. Choi, arXiv:hep-ph/0511162.

[11] B. C. Allanach, F. Quevedo and K. Suruliz, JHEP 0604, 040 (2006) [arXiv:hep-ph/0512081].

[12] J. P. Conlon and F. Quevedo, JHEP 0606, 029 (2006) [arXiv:hep-th/0605141].

[13] G. Curio and V. Spillner, arXiv:hep-th/0606047.

[14] J. P. Conlon, S. S. Abdussalam, F. Quevedo and K. Suruliz, arXiv:hep-th/0610129.

[15] M. Berg, M. Haack and E. Pajer, arXiv:0704.0737 [hep-th].

[16] A. Misra and P. Shukla, arXiv:0707.0105 [hep-th].

cq [17] J. P. Conlon and F. Quevedo, arXiv:0705.3460 [hep-ph].

eff-sugra [18] T. W. Grimm and J. Louis, Nucl. Phys. B 699, 387 (2004) [arXiv:hep-th/0403067]. 
BKQ [19] C. P. Burgess, R. Kallosh and F. Quevedo, JHEP 0310 (2003) 056 [arXiv:hep-th/0309187].

hepth0404116

CMQS

hepth0611332

eva-ds

hepth0404134

louis

CCQPaper

bim

choi

hepth0408054

BBHL

cksaq

p11169

[20] F. Denef and M. R. Douglas, JHEP 0405, 072 (2004) [arXiv:hep-th/0404116].

[21] D. Cremades, M. P. Garcia del Moral, F. Quevedo and K. Suruliz, arXiv:hep-th/0701154.

[22] A. Westphal, JHEP 0703 (2007) 102 [arXiv:hep-th/0611332].

[23] A. Saltman and E. Silverstein, JHEP 0411 (2004) 066 [arXiv:hep-th/0402135].

[24] D. Lust, P. Mayr, R. Richter and S. Stieberger, Nucl. Phys. B 696, 205 (2004) [arXiv:hep-th/0404134].

[25] H. Jockers and J. Louis, Nucl. Phys. B 705 (2005) 167 [arXiv:hep-th/0409098].

[26] J. P. Conlon, D. Cremades and F. Quevedo, arXiv:hep-th/0609180.

[27] V. S. Kaplunovsky and J. Louis, Phys. Lett. B 306 (1993) 269 [arXiv:hep-th/9303040]; A. Brignole, L. E. Ibáñez and C. Munoz, Nucl. Phys. B 422 (1994) 125 [Erratum-ibid. B 436 (1995) 747] [arXiv:hep-ph/9308271].

[28] K. Choi, A. Falkowski, H. P. Nilles and M. Olechowski, Nucl. Phys. B 718, 113 (2005) [arXiv:hep-th/0503216].

[29] V. Balasubramanian and P. Berglund, JHEP 0411 (2004) 085 [arXiv:hep-th/0408054].

[30] K. Becker, M. Becker, M. Haack and J. Louis, JHEP 0206 (2002) 060 [arXiv:hep-th/0204254].

[31] J. P. Conlon, C. H. Kom, K. Suruliz, B. C. Allanach and F. Quevedo, arXiv:0704.3403 [hep-ph].

[32] P. Candelas, A. Font, S. H. Katz and D. R. Morrison, Nucl. Phys. B 429 (1994) 626 [arXiv:hep-th/9403187]; D. E. Diaconescu and C. Romelsberger, Nucl. Phys. B 574 (2000) 245 [arXiv:hep-th/9910172]; F. Denef, M. R. Douglas and B. Florea, JHEP 0406 (2004) 034 [arXiv:hep-th/0404257]. 